\title{
Allocation of Decision-Making Authority with Principal's Reputation Concerns
}

\author{
Yasunari Tamada ${ }^{\dagger} \quad$ Tsung-Sheng Tsai ${ }^{\ddagger}$ \\ Keio University Academia Sinica
}

This version: March 2004

\begin{abstract}
This paper analyzes the allocation of decision-making authority when the principal has reputation concerns. The principal can either keep the authority and consult the agent (an expert), or delegate the authority to the agent; however, the outside evaluator cannot observe the allocation of authority. Hence, delegation can provide a way to manipulate the principal's ex post reputation. In general, the principal keeps the authority too often when she has the opportunity of delegation. When the evaluator believes that the agent may make the decision sometimes, the principal has less incentive to make the right decisions.
\end{abstract}

Keywords: Delegation, Reputation Concerns, Cheap Talk

JEL classification: D23, D82, L14

\footnotetext{
${ }^{\dagger}$ Department of Economics, Keio University, 2-15-45 Mita, Minato-ku, Tokyo, 108-8345, Japan. Email: tamada@econ.keio.ac.jp.

${ }^{\ddagger}$ Institute of Economics, Academia Sinica, 128, Sec. 2, Yan-jiou-yuan Rd, Nankang, Taipei, 11529, Taiwan. Email: tstsai@econ.sinica.edu.tw.
} 


\section{Introduction}

Delegation of the decision-making authority from the decision makers to their subordinates has been an important issue in organization theory. For example, in Congress, where policy is made, by legislature or through delegation to the bureaucrats, has a significant impact on policy outcomes. ${ }^{1}$ In the internal organization of firm, the allocation of decision-making authority between a CEO and divisionmanagers, or between a division manager and her subordinate is important in firm's performance.

One conventional explanation of why the principal delegates the authority to the agent is to take advantages of the agent's expertise and reduce her workload. However, due to conflict interests and asymmetric information, the control cannot be perfect. Therefore, as argued in Aghion and Tirole (1997), the principal thus faces a trade-off between a loss of control and a loss of information: when the principal delegates the authority, it provides the agent the incentive to acquire information, which results in better quality of information; however, differences in preferences between the principal and the agent lead to biased decisions. When communication between the principal and the agent is possible, Dessein (2002) shows that delegation can be always better than informative communication. Harris and Raviv (2001) consider the case where the principal also has private information and conclude that the principal prefers delegation to communication when the agent's information is relatively important. Sometimes, the principal may delegate more often even when

\footnotetext{
${ }^{1}$ The literature has been paying much more attention on the optimal "degree of delegation" in controlling the agency problem. For example, Epstein and O'Halloran (1994) show that when Congress has appropriate administrative procedures, which include ex ante delegation and ex post veto, it will delegate a large degree of authority, regardless of their differences in policy preferences. Epstein and O'Halloran (1999) observe that Congress keeps authority the most in issues of budget, rules, ways and means, but delegates the authority to the executive the most in areas of agricultural and public works, and armed services. Gailmard (2002) introduces the bureaucrat's subversion into Epstein and O'Halloran's (1999) model, which leads to less delegation.
} 
the agent is more biased.

In this paper, we propose another reason for the principal to delegate the decisionmaking authority; namely, her reputation concerns. It matters when the outside evaluator cannot observe the principal's preference on making the appropriate decisions, nor the allocation of authority. That is, the outside evaluator can only conjecture about the principal's type and who makes the decision from the decision outcomes. Therefore, the principal faces a trade-off: she can either keep the authority and choose her ideal action, or she can increase her ex post reputation and make use of the agent's expertise by delegation.

When the allocation of authority is fixed or beyond the principal's control, from the evaluator's view, the agent's discretion is better than the principal's discretion, simply because the agent has a better chance to make the right decisions. That is, delegation is always better for the evaluator. On the other hand, when the principal can choose the allocation of authority, and the evaluator cannot observe it, the principal can manipulate her ex post reputation by her delegation policy. In general, the principal keeps the authority too often when delegation can be an option. When the evaluator believes that the agent may make the decision sometimes, the principal has less incentive to choose the right decision.

Reputation concern has different effects on delegation policy for the principal of different types. When reputation is less important, the biased principal would like to keep the authority and make her ideal policy. The good one is indifferent between keeping the authority and delegation, because in both cases, she prefers correct decisions. When reputation is important, the biased principal prefers delegation because it is costly to make the biased policy. The good one prefers keeping the authority, and makes a different policy to distinguish from the biased one. When reputation concern is intermediate, then it is possible that both types delegate sometimes. Since delegation is always better for the evaluator, the case is the best for the evaluator. 
Our work is closed to Levy (2000), although delegation is not an option in her model, in that the principal cares about both the appropriate decisions and her reputation, and she can consult the agent before making the decisions. Levy shows that the more competent principal, the less she consults: in order to show the evaluator that she is better than the agent, she takes the actions contradicting the agent's advices, and thus in equilibrium, it results in too much contradictions, i.e., anti-herding. $^{2}$

In the literature on contract or organization theory, the importance of observability of contracts to the principal's reputation concerns has not yet been explored much. ${ }^{3}$ In politicians/bureaucrats relationship, politicians care about re-election. If voters cannot observe who makes the decisions, then politicians can manipulate their reputation by their delegation policy. Our results imply that politicians tend to delegate the authority of making some unpopular policies (in that politicians would look biased if they made such policies), for example, pork barrel programs, and shift the blame to the bureaucrats, as long as the voters believe that politicians are not responsible for all the decision-making.

The rest of paper is organized as follows. Section 2 introduces the model. Section 3 discusses the equilibrium under the principal's discretion and under the agent's discretion, when the regime is fixed. Section 4 analyzes the principal's optimal allocation of authority. Section 5 concludes the paper.

\footnotetext{
${ }^{2}$ Other researchers care about the agent's career concerns, which has been recognized as a cause for information loss and inefficient decision making. See, for example, Morris (2001), Ottaviani and Sørensen (2002), and Scharfstein and Stein (1990).

${ }^{3}$ In the example of labor market, Andersson (2002) considers the case where the labor market is not able to observe the contracts between workers and their (former) employers, but only workers' wages. However, with workers' career concerns, they work too much in order to be evaluated higher in the market. With observable contracts, on the other hand, career concerns have no effects on workers' incentives in efforts.
} 


\section{Model}

There are three players in the communication/delegation game: a principal (she), an agent (he), and an outside evaluator. For example, we can interpret the principal as a politician or a CEO, the agent as a bureaucrat or a division manager, and the evaluator as the median voter or the labor market.

The principal can either keep the authority and consult the agent, or delegate the decision-making authority to the agent. The party who has the formal authority chooses an action, $x \in\{0,1\}$. However, the outcomes also depend on the states of the world, $\theta \in\{0,1\}$, which are unobservable to the principal. We assume that each state occurs with probability $1 / 2$. The appropriate action is made if $x=\theta$, where the appropriateness of actions is evaluated by the evaluator's point of view. While the principal has no further information about $\theta$, the agent can perfectly observe it. That is, the agent is an expert and informational superior to the principal. On the other hand, the evaluator can observe the realized $\theta$ and the implemented $x$, although he may not be able to observe the allocation of authority, nor players' types.

\section{Information}

Both the principal and the agent are either good (unbiased) or biased, in that the good type party prefers the appropriate decisions, and the biased type always prefers $x=1$. Types are private information, so that the principal and the agent know their own type, but not the other party's type. The evaluator is also uncertain about both the principal's and the agent's type. The common prior beliefs are that the principal is good with probability $\mu_{p} \in(0,1)$ and biased with probability $1-\mu_{p}$, and that the agent is good with probability $\mu_{a} \in(0,1)$ and biased with probability $1-\mu_{a}$. Thus, $\mu_{p}$ can be interpreted as the principal's ex ante reputation. 


\section{Preferences}

We assume that the principal receives the utility from the outcome and her ex post reputation (i.e., the evaluator's ex post beliefs about the principal being the good type). Namely, the good principal's utility function is

$$
U_{p}^{G}\left(x, \theta, \hat{\mu}_{p}\right)=-|x-\theta|+\gamma \hat{\mu}_{p}
$$

and the biased principal, who always prefers $x=1$, has the utility function

$$
U_{p}^{B}\left(x, \theta, \hat{\mu}_{p}\right)=-|x-1|+\gamma \hat{\mu}_{p}
$$

where $\hat{\mu}_{p}$ denotes the principal's ex post reputation and $\gamma>0$ is its relative importance.

The agent has no reputation concerns. Thus, the good agent's utility function is

$$
U_{a}^{G}(x, \theta)=-|x-\theta|,
$$

and the biased agent's utility function is

$$
U_{a}^{B}(x, \theta)=-|x-1|
$$

Finally, the evaluator's utility function is $-|x-\theta|$.

\section{Allocation of Authority}

We assume that the principal is unable to commit to any implementation function. More precisely, information about $\theta$ is soft so that the agent cannot verify it, and the agent cannot announce his type in a credible way through the contracts, so that the principal cannot elicit the agent's private information via revelation mechanisms, and thus, the agent is unconstrained in sending any message. The only variable the principal can specify in the contracts is the allocation of authority (control rights). 
Initially, the principal keeps the control. Then, she can choose either the principal's discretion or the agent's discretion.

Under the principal's discretion, the principal keeps the decision-making authority. The agent is requested to send a message $m \in\{0,1\}$ to the principal about his private information $\theta$. Then the principal chooses an action based on the agent's message; that is, the principal and the agent play a cheap-talk game.

Under the agent's discretion, the principal delegates the control right to the agent, and thus the agent has the formal authority to make the decisions. In this case, the principal can commit to never intervene in the agent's decisions.

\section{Timing}

The timing of the game is as follows:

1. The principal chooses the allocation of decision-making authority.

2. The agent observes $\theta$ and sends a message $m$.

3. Given the allocation of authority, the party who has the formal authority chooses $x$.

4. The evaluator observes the realized $\theta$ and $x$, and updates the principal's reputation (from $\mu_{p}$ to $\hat{\mu}_{p}$ ).

\section{Benchmark: Fixed Discretion}

We first consider the benchmark case where the allocation of authority is fixed, in other words, the principal cannot choose the delegation policy. Instead, for example, it is the evaluator who can choose the allocation of authority and commit to the regime. In this case, the principal can only manipulate her ex post reputation by the 
policy she chooses. We will show that the agent's discretion is always better than the principal's discretion from the evaluator's view. However, as will be shown in the next section, when principal can choose the allocation of authority, with positive probability, the principal will keep the authority in equilibrium.

\subsection{Principal's Discretion}

First, we consider the principal's discretion. The principal and the agent play a cheap-talk game in which the agent sends a message to the principal, and the principal makes the decision after listening to the agent's message. The evaluator, however, cannot observe the agent's advices.

Here, we only focus on an informative (nonbabbling) equilibrium in the cheap talk game. ${ }^{4}$ In this equilibrium, the good principal always chooses $x=0$ if $m=0$. However, as in Morris (2001), because of the reputation concern, there exist some equilibria in which the good principal may make a wrong decision in order to be distinguished from the biased type; that is, she chooses $x=0$ when she believes that $\theta=1$ happens. On the other hand, the biased principal always chooses $x=1$ if $m=1$, but she may choose $x=0$ when she believes that $\theta=0$ happens, in order to mimic the good type and gain some ex post reputation. To assure that there exists such an equilibrium, we make one assumption:

Assumption $1 \frac{1}{1-\mu_{p}}>\gamma$.

Assumption 1 is satisfied if the principal is good with sufficiently large probability and/or the principal's reputation concern is not too large. The following

\footnotetext{
${ }^{4}$ As in the standard cheap-talk game as in Crawford and Sobel (1982), there exist babbling equilibria in which messages do not convey any information. For example, if the agent of both types always send $m=1$ regardless of $\theta$, then the principal learns nothing from the message, and simply puts probability $1 / 2$ to each state. Given this principal's belief, both types have no incentives to deviate from their uninformative announcements.
} 
proposition characterizes the properties of this equilibrium.

\section{Proposition 1}

Suppose that Assumption 1 holds. Under the principal's discretion, there exists an equilibrium which satisfies,

1. The good agent always sends $m=\theta$, and the biased agent always sends $m=1$.

2. If $m=0$, then the good principal always chooses $x=0$, and if $m=1$, she chooses $x=1$ with probability $\nu_{G}^{*} \in[0,1]$.

3. If $m=1$, then the biased principal chooses $x=1$, and if $m=0$, the biased principal always chooses $x=0$ with probability $\nu_{B}^{*} \in[0,1)$.

Proof. See the Appendix.

Let $\hat{\mu}_{p}\left(x, \theta, \nu_{G}^{*}, \nu_{B}^{*}\right)$ be the principal's ex post reputation when the evaluator observes $(x, \theta)$. We have

$$
\begin{gathered}
\hat{\mu}_{p}\left(0,0, \nu_{G}, \nu_{B}\right)=\frac{\mu_{p}\left[\mu_{a}+\left(1-\mu_{a}\right)\left(1-\nu_{G}\right)\right]}{\mu_{p}\left[\mu_{a}+\left(1-\mu_{a}\right)\left(1-\nu_{G}\right)\right]+\left(1-\mu_{p}\right) \mu_{a} \nu_{B}}, \\
\hat{\mu}_{p}\left(1,1, \nu_{G}, \nu_{B}\right)=\frac{\mu_{p} \nu_{G}}{\mu_{p} \nu_{G}+\left(1-\mu_{p}\right)}, \\
\hat{\mu}_{p}\left(1,0, \nu_{G}, \nu_{B}\right)=\frac{\mu_{p}\left(1-\mu_{a}\right) \nu_{G}}{\mu_{p}\left(1-\mu_{a}\right) \nu_{G}+\left(1-\mu_{p}\right)\left[\mu_{a}\left(1-\nu_{B}\right)+\left(1-\mu_{a}\right)\right]}, \\
\hat{\mu}_{p}\left(0,1, \nu_{G}, \nu_{B}\right)=1 .
\end{gathered}
$$

According to equations (5) - (8), we have the following result: 


\section{Corollary 1}

$\hat{\mu}_{p}\left(0,1, \nu_{G}, \nu_{B}\right) \geq \hat{\mu}_{p}\left(0,0, \nu_{G}, \nu_{B}\right) \geq \mu_{p} \geq \hat{\mu}_{p}\left(1,1, \nu_{G}, \nu_{B}\right) \geq \hat{\mu}_{p}\left(1,0, \nu_{G}, \nu_{B}\right)$.

For the biased principal, she has a tendency to choose $x=1$; however, because of reputation concern, she may choose $x=0$ when $m=0$ with positive probability $\nu_{B}^{*}$ to pretend as a good one, since $\hat{\mu}_{p}\left(0,0, \nu_{G}, \nu_{B}\right)$ is decreasing in $\nu_{B}$ and $\hat{\mu}_{p}\left(1,0, \nu_{G}, \nu_{B}\right)$ is non-decreasing in $\nu_{B}$. Although the evaluator tends to regard the principal as the biased one when he observes $(x, \theta)=(1,0), \nu_{B}^{*}=1$ cannot be a part of the equilibrium, because it implies that the evaluator would not be able to distinguish the good type from the biased one, and thus the biased type can choose $x=1$ without damaging her reputation. Therefore, $\nu_{B}^{*}<1$. On the other hand, the good principal may choose $x=0$ when $m=1$ in order to show that she is unbiased, since $\hat{\mu}_{p}\left(1,1, \nu_{G}, \nu_{B}\right)$ is increasing in $\nu_{G}$, although it causes some loss for her from making such a wrong decision. As for the agent's behavior, the good agent always sends $m=\theta$ as long as the principal regards that the agent's messages are informative. The biased agent has no incentive to send $m=0$ since he can induce $x=1$ by sending $m=1$. Thus, communication between the principal and the agent is informative but noisy.

\section{Corollary 2}

$\nu_{G}^{*}$ and $\nu_{B}^{*}$ are non-decreasing in $\mu_{a}$.

The biased principal prefers $x=0$ if she could jeopardize her ex post reputation $\hat{\mu}_{p}\left(1,0, \nu_{G}^{*}, \nu_{B}^{*}\right)$ too much when she chooses $x=1$. Since $\hat{\mu}_{p}\left(1,0, \nu_{G}^{*}, \nu_{B}^{*}\right)$ decreases in $\mu_{a}$, the higher $\mu_{a}$, the more likely that the evaluator thinks that this wrong decision is made by the biased principal. Therefore, in order to keep a good reputation, the biased principal has more incentives to keep accountable. The case of the good principal is similar.

Accordingly, under the principal's discretion (denoted by $P$ ), the expected utility 
of the good principal in equilibrium is

$$
\begin{aligned}
E U_{p}^{G}(P)= & \frac{1}{2}\left\{\mu_{a} \gamma \hat{\mu}_{p}\left(0,0, \nu_{G}^{*}, \nu_{B}^{*}\right)\right. \\
& \left.+\left(1-\mu_{a}\right)\left[\left(1-\nu_{G}^{*}\right) \gamma \hat{\mu}_{p}\left(0,0, \nu_{G}^{*}, \nu_{B}^{*}\right)+\nu_{G}^{*}\left(-1+\gamma \hat{\mu}_{p}\left(1,0, \nu_{G}^{*}, \nu_{B}^{*}\right)\right)\right]\right\} \\
& +\frac{1}{2}\left\{\nu_{G}^{*} \gamma \hat{\mu}_{p}\left(1,1, \nu_{G}^{*}, \nu_{B}^{*}\right)+\left(1-\nu_{G}^{*}\right)\left(-1+\gamma \hat{\mu}_{p}\left(0,1, \nu_{G}^{*}, \nu_{B}^{*}\right)\right)\right\}
\end{aligned}
$$

and that of the biased principal is

$$
\begin{aligned}
E U_{p}^{B}(P)= & \frac{1}{2}\left\{\mu_{a}\left[\nu_{B}^{*}\left(-1+\gamma \hat{\mu}_{p}\left(0,0, \nu_{G}^{*}, \nu_{B}^{*}\right)\right)+\left(1-\nu_{B}^{*}\right) \gamma \hat{\mu}_{p}\left(1,0, \nu_{G}^{*}, \nu_{B}^{*}\right)\right]\right. \\
& \left.+\left(1-\mu_{a}\right) \gamma \hat{\mu}_{p}\left(1,0, \nu_{G}^{*}, \nu_{B}^{*}\right)\right\}+\frac{1}{2} \gamma \hat{\mu}_{p}\left(1,1, \nu_{G}^{*}, \nu_{B}^{*}\right)
\end{aligned}
$$

\subsection{Agent's Discretion}

When the agent has the decision-making authority, he can choose his ideal decisions at will by using his private information. Obviously, the good agent chooses $x=\theta$, and the biased agent always chooses $x=1$ regardless of $\theta$.

Thus, under the agent's discretion (denoted by $A$ ), the good principal's expected utility in equilibrium is

$$
E U_{p}^{G}(A)=-\frac{1}{2}\left(1-\mu_{a}\right)+\gamma \mu_{p}
$$

and the biased principal's expected utility is

$$
E U_{p}^{B}(A)=-\frac{1}{2} \mu_{a}+\gamma \mu_{p}
$$

Note that the principal's reputation is not updated since, under the agent's discretion, the evaluator has no access to any information about the principal's type.

\subsection{Principal's Discretion vs. Agent's Discretion}

So far, we assume that the allocation of authority is fixed or beyond the principal's control. From the evaluator's view, the optimal decision is $x=\theta$. If $\theta=1$, 
$x=1$ is implemented for sure under the agent's discretion, but with probability $\mu_{p} \nu_{G}^{*}+\left(1-\mu_{p}\right)$ under the principal discretion. If $\theta=0, x=0$ is implemented with probability $\mu_{a}$ under the agent's discretion, and with probability $\mu_{a}\left(\mu_{p}+\left(1-\mu_{p}\right) \nu_{B}^{*}\right)$ under the principal's discretion. Since $\nu_{B}^{*}<1$, the agent's discretion is always better than the principal's discretion for the evaluator. Thus, if it is possible to fix the regime, the evaluator prefers the agent's discretion. The reason for this result is that there are double-sided biases under the principal's discretion (from both the agent's bias in communication and the principal's bias in decision-making), while there is only one-sided bias under the agent's discretion. The result can be summarized in the following proposition.

\section{Proposition 2}

Under fixed discretion, the evaluator prefers the agent's discretion.

\section{Principal's Delegation Policy}

If the principal has the power to decide the allocation of authority, and the evaluator cannot observe it, the evaluator must guess who actually makes the decisions. In equilibrium, the evaluator's conjucture must be consistent with the principal's choice of the allocation.

Let $\alpha^{G}$ (resp. $\alpha^{B}$ ) be the probability that the good (resp. biased) principal keeps the decision-making authority. Thus, given the evaluator's beliefs, $\hat{\alpha}^{G}$ and $\hat{\alpha}^{B}$, he believes that the decision-making authority belongs to the principal with probability $\mu_{p} \hat{\alpha}^{G}+\left(1-\mu_{p}\right) \hat{\alpha}^{B}$, and to the agent with probability $1-\left(\mu_{p} \hat{\alpha}^{G}+\left(1-\mu_{p}\right) \hat{\alpha}^{B}\right)$. We will still focus on the informative equilibrium as in the last section.

In the last period, the evaluator observes $\theta$ and $x$, and forms his beliefs on $\alpha^{G}, \alpha^{B}$, and $\nu_{G}, \nu_{B}$. As before, the good agent chooses $x=\theta$ and the biased agent chooses 
$x=1$ under the agent's discretion. Therefore, the principal's ex post reputations $\hat{\mu}_{p}\left(x, \theta, \nu_{G}, \nu_{B}, \hat{\alpha}^{G}, \hat{\alpha}^{B}\right) \equiv \tilde{\mu}_{p}\left(x, \theta, \nu_{G}, \nu_{B}\right)$ become

$$
\begin{gathered}
\tilde{\mu}_{p}\left(0,0, \nu_{G}, \nu_{B}\right)=\frac{\mu_{p}\left[\hat{\alpha}^{G}\left(\mu_{a}+\left(1-\mu_{a}\right)\left(1-\nu_{G}\right)\right)+\left(1-\hat{\alpha}^{G}\right) \mu_{a}\right]}{\mu_{p}\left[\hat{\alpha}^{G}\left(\mu_{a}+\left(1-\mu_{a}\right)\left(1-\nu_{G}\right)\right)+\left(1-\hat{\alpha}^{G}\right) \mu_{a}\right]+\left(1-\mu_{p}\right) \mu_{a}\left[\hat{\alpha}^{B} \nu_{B}+\left(1-\hat{\alpha}^{B}\right)\right]} \\
\tilde{\mu}_{p}\left(1,1, \nu_{G}, \nu_{B}\right)=\frac{\mu_{p}\left[\hat{\alpha}^{G} \nu_{G}+\left(1-\hat{\alpha}^{G}\right)\right]}{\mu_{p}\left[\hat{\alpha}^{G} \nu_{G}+\left(1-\hat{\alpha}^{G}\right)\right]+\left(1-\mu_{p}\right)} \\
\tilde{\mu}_{p}\left(1,0, \nu_{G}, \nu_{B}\right)=\frac{\mu_{p}\left[\hat{\alpha}^{G}\left(1-\mu_{a}\right) \nu_{G}+\left(1-\hat{\alpha}^{G}\right)\left(1-\mu_{a}\right)\right]}{\mu_{p}\left[\hat{\alpha}^{G}\left(1-\mu_{a}\right) \nu_{G}+\left(1-\hat{\alpha}^{G}\right)\left(1-\mu_{a}\right)\right]+\left(1-\mu_{p}\right)\left[\hat{\alpha}^{B}\left(1-\mu_{a} \nu_{B}\right)+\left(1-\hat{\alpha}^{B}\right)\left(1-\mu_{a}\right)\right]} \\
\tilde{\mu}_{p}\left(0,1, \nu_{G}, \nu_{B}\right)=1
\end{gathered}
$$

\subsection{The Biased Principal}

First, consider the biased principal's decision making under the principal's discretion at period 3. Let $\nu_{B}^{* *}$ be the equilibrium probability that the biased principal chooses $x=0$ when $m=0$ for any given $\left(\hat{\alpha}^{G}, \hat{\alpha}^{B}\right)$. We have the following lemma:

Lemma 1 Given the evaluator's beliefs $\left(\hat{\alpha}^{G}, \hat{\alpha}^{B}\right), \nu_{B}^{* *} \leq \nu_{B}^{*}$ must hold. Furthermore, if $\hat{\alpha}^{B}<1$ and $\nu_{B}^{*} \in(0,1)$, then $\nu_{B}^{* *}<\nu_{B}^{*}$.

Proof. See the Appendix.

Lemma 1 says that if the evaluator believes that the biased principal may delegate with some probability, i.e., $\hat{\alpha}^{B}<1$, the probability that the biased principal chooses $x=0$ when $m=0$ cannot be greater than that of the case where the evaluator knows that the principal has the authority. The reason is simple. The incentive for the biased agent to choose $x=0$ comes from her reputation concerns. From (13) and (15), one can check that the smaller $\hat{\alpha}^{B}$, the smaller $\tilde{\mu}_{p}\left(0,0, \nu_{G}, \nu_{B}\right)$ and the 
larger $\tilde{\mu}_{p}\left(1,0, \nu_{G}, \nu_{B}\right)$. In this case, the biased principal tends to choose $x=1$ even when $m=0$. Since $\nu_{B}^{*}$ corresponds to the case $\hat{\alpha}^{B}=1, \nu_{B}^{* *} \leq \nu_{B}^{*}$ must be the case.

Now, let us consider the biased principal's delegation policy. Let $\alpha^{B *}$ be the probability in equilibrium that the biased principal keeps decision-making authority. We can show the following proposition.

\section{Proposition 3}

$\alpha^{B *} \in(0,1]$. Moreover, $\alpha^{B *}=1$ if and only if $\nu_{B}^{* *}=0$.

The biased principal faces a trade-off between her reputation concerns and her preferred decisions. Consider the case in which the evaluator believes that $\hat{\alpha}^{B}=0$, that is, the agent has the authority. In this case, the biased principal can deviate to keeping the authority and choosing $x=1$ without damaging her reputation, since the evaluator believes that the decision is made by the agent. Thus, the biased principal has the incentive to keep the authority with some positive probability. On the other hand, suppose that the evaluator believes that $\hat{\alpha}^{B}=1$, that is, the principal makes the decisions. In this case, the biased principal could deteriorate her reputation if she chooses her ideal action $x=1$. Therefore, if the reputation is sufficiently important, she should choose $\alpha^{B}<1$. Otherwise, she would rather keep the authority and always choose her preferred policy, $x=1$, when reputation is not a crucial concern.

\subsection{The Good Principal}

Next, consider the good principal's delegation policy. Let $\nu_{G}^{* *}$ be the equilibrium probability that the good principal chooses $x=1$ when $m=1$ given the evaluator's beliefs $\left(\hat{\alpha}^{G}, \hat{\alpha}^{B}\right)$. We have the the following result: 


\section{Proposition 4}

1. Given any $\left(\hat{\alpha}^{G}, \hat{\alpha}^{B}\right), \alpha^{G *} \in[0,1]$ can be an equilibrium.

2. If $\nu_{G}^{* *}=0$, then $\alpha^{G *}=1$. If $\nu_{G}^{* *} \in(0,1)$, then $\alpha^{G *} \in(0,1)$. If $\nu_{G}^{* *}=1$, then she is indifferent.

3. $\nu_{G}^{* *} \leq \nu_{G}^{*}$.

Proof. See the Appendix.

Similar to the biased principal, when $x=0$ is always the best for the good principal at $m=1$, she will keep the authority. Otherwise, with less important reputation concern, she prefers delegation sometimes and behaves more accountable.

\subsection{The Effect of Reputation Concerns}

From Proposition 3 and 4, because of reputation concerns, we can see that in general, the principal keep too much authority. However, reputation concerns have different effects delegation policy for the principal of different types. When $\gamma$ is small, the biased principal prefers keeping the authority and makes her ideal policy. The good one is in fact indifferent between keeping the authority and delegation, because in both cases, it is better to make the correct decisions. When $\gamma$ is large, the biased principal prefers delegation because it is costly to make the biased policy. The good one prefers keeping the authority, and makes policy $x=0$ in order to be distinguished from the biased one. When $\gamma$ is intermediate, then it is possible that both types delegate sometimes. Since delegation is always better for the evaluator, the case is best for the evaluator.

Under principal's discretion in the fixed regime case, the only tool that the principal can apply is making the right decisions. In contrast, when the evaluator believes that the agent may make the decision sometimes, the principal has another 
way to manipulate her ex post reputation. Since the evaluator cannot observe who indeed makes the decision, the principal would take this advantage and has more incentives to make the policy she prefers (i.e., $x=1$ is made by the biased type and $x=0$ by the good type more often), and blames on the agent for wrong decisions.

\section{Concluding Remarks}

In this paper, we investigate how the principal strategically allocates the decisionmaking authority when she has reputation concerns. When the agent is informational superior to the principal, the basic trade-off for the principal is between implementing the ideal decisions and maintaining her ex post reputation. We argue that delegation can provide a way for the principal to manipulate her ex post reputation. In general, the principal keeps the authority too often when she has the opportunity of delegation. When the evaluator believes that the agent may make the decision sometimes, the principal has less incentive to follow the agent's advices and would like to make her preferred policy more often.

Our results depend upon some restrictive assumptions. First we limit our attention to an informative equilibrium in which the good (biased) principal always follows the agent's recommendations when $m=0$ (when $m=1$ ). A more general analysis on optimal delegation policy in all equilibria is called for. In Appendix B, we reconsider Proposition 1 by including all possible cases. Second, our results depend upon the unobservability of the allocation of authority. For example, if the evaluator not only can observe what decisions have been made, but also who makes them, then the evaluator can evaluate principal's ability by their allocation policy. Since delegation to the agent appears to be a bad signal for the principal, the biased principal may also want to keep the authority. Although the formation of the delegation policy in this paper is somehow restricted, we extract the effect of principal's 
reputation concerns on the allocation of authority, which has been neglected very much so far in the large literatures on organization theory.

\section{References}

Andersson, F. (2002), "Career Concerns, Contracts, and Effort Distortion," Journal of Labor Economics 20, 42-58.

Aghion, P. and J. Tirole (1997), "Formal and Real Authority in Organizations," Journal of Political Economy 105, 1-29.

Crawford V. and J. Sobel (1982), "Strategic Information Transmission," Econometrica 50, 1434-1451.

Dessein, W. (2002), "Authority and Communication in Organizations," Review of Economic Studies 69, 811-838.

Esptein, D. and S. O'Halloran (1994), "Administrative Procedures, Information, and Agency Discretion," American Journal of Political Science 38, 697-722.

Esptein, D. and S. O'Halloran (1999), Delegation Power: A Transaction Cost Politics Approach to Policy Making Under Separate Powers. Cambridge: Cambridge University Press.

Gailmard, S. (2002), "Expertise, Subversion, and Bureaucratic Discretion," Journal of Law, Economics, and Organization 18, 536-555.

Harris, M. and A. Raviv (2001), "Allocation of Decision-Making Authority," mimeo.

Levy, G. (2000), "Strategic Consultation and Strategic Recruiting in the Presence of Career Concerns," mimeo. 
Morris, S. (2001), "Political Correctness," Journal of Political Economy 109, 231265.

Ottaviani, M. and P. Sørensen (2002), "Professional Advices: The Theory of Reputational Cheap Talk," mimeo.

Scharfstein, D. and J. Stein (1990), "Herd Behavior and Investment," American Economic Review 80, 465-479. 


\section{Appendix A}

\section{Proof of Proposition 1.}

To prove Proposition 1, we make several claims as follows.

\section{Claim 1}

Suppose that the good agent always sends $m=\theta$ and the biased agent sends $m=1$ always. Also, suppose that the good principal always chooses $x=0$ if $m=0$, and chooses $x=1$ with probability $\nu_{G}^{*} \in[0,1]$ if $m=1$. Then, the biased principal always chooses $x=1$ if $m=1$, and chooses $x=0$ with probability $\nu_{B}^{*} \in[0,1)$ if $m=0$.

Proof.

If the biased principal chooses $x=1$ when $m=1$, she obtains $\gamma \hat{\mu}_{p}\left(1,1, \nu_{G}, \nu_{B}\right)$. If she instead chooses $x=0$, she gets $-1+\gamma \hat{\mu}_{p}\left(0,1, \nu_{G}, \nu_{B}\right)$. If Assumption 1 is satisfied, there exists a $\nu_{G}^{*}$ such that

$$
\gamma \hat{\mu}_{p}\left(1,1, \nu_{G}, \nu_{B}\right)>-1+\gamma \hat{\mu}_{p}\left(0,1, \nu_{G}, \nu_{B}\right)=-1+\gamma
$$

and thus she chooses $x=1$.

Suppose $m=0$. If she implements $x=1$, then her expected utility becomes $\gamma \hat{\mu}_{p}\left(1,0, \nu_{G}, \nu_{B}\right)$. If she implements $x=0$, her expected utility is $-1+$ $\gamma \hat{\mu}_{p}\left(0,0, \nu_{G}, \nu_{B}\right)$. If $\nu_{B}=1$, it must be $-1+\gamma \hat{\mu}_{p}\left(0,0, \nu_{G}, \nu_{B}\right)>\gamma \hat{\mu}_{p}\left(1,0, \nu_{G}, \nu_{B}\right)$. In this case, $\hat{\mu}_{p}\left(1,1, \nu_{G}, \nu_{B}\right)=\hat{\mu}_{p}\left(1,0, \nu_{G}, \nu_{B}\right)$. According to (17), it implies that $\hat{\mu}_{p}\left(0,0, \nu_{G}, \nu_{B}\right)>\hat{\mu}_{p}\left(0,1, \nu_{G}, \nu_{B}\right)$, which is not possible. Therefore, it must be $\nu_{B}<1$ and so

$$
-1+\gamma \hat{\mu}_{p}\left(0,0, \nu_{G}, \nu_{B}\right) \leq \gamma \hat{\mu}_{p}\left(1,0, \nu_{G}, \nu_{B}\right)
$$




\section{Claim 2}

Suppose that the good agent always sends $m=\theta$ and the biased agent always sends $m=1$. Furthermore, suppose that the biased principal chooses $x=1$ when $m=1$, and $x=0$ with probability $\nu_{B}^{*} \in[0,1)$ when $m=0$. Then, the good principal always chooses $x=0$ if $m=0$, and chooses $x=1$ with probability $\nu_{G}^{*} \in[0,1]$ if $m=1$.

\section{Proof.}

When $m=0$, if the good principal chooses $x=0$, she obtains $\gamma \hat{\mu}_{p}\left(0,0, \nu_{G}, \nu_{B}\right)$. If she instead chooses $x=1$, she gets $-1+\gamma \hat{\mu}_{p}\left(1,0, \nu_{G}, \nu_{B}\right)$. Thus, regardless of $\nu_{B}$ and $\gamma$, she chooses $x=0$ based on Corollary 1 .

When $m=1$, if she implements $x=0$, then her expected utility becomes $\frac{1}{2}\left[-1+\gamma \hat{\mu}_{p}\left(0,1, \nu_{G}, \nu_{B}\right)\right]+\frac{1}{2}\left(1-\mu_{a}\right) \gamma \hat{\mu}_{p}\left(0,0, \nu_{G}, \nu_{B}\right)$. If she chooses $x=1$ instead, her expected utility is $\frac{1}{2} \gamma \hat{\mu}_{p}\left(1,1, \nu_{G}, \nu_{B}\right)+\frac{1}{2}\left(1-\mu_{a}\right)\left[-1+\gamma \hat{\mu}_{p}\left(1,0, \nu_{G}, \nu_{B}\right)\right]$. Therefore, $\nu_{G}^{*}<1$ if

$$
\begin{aligned}
& {\left[-1+\gamma \hat{\mu}_{p}\left(0,1, \nu_{G}, \nu_{B}\right)\right]+\left(1-\mu_{a}\right) \gamma \hat{\mu}_{p}\left(0,0, \nu_{G}, \nu_{B}\right) } \\
& \geq \gamma \hat{\mu}_{p}\left(1,1, \nu_{G}, \nu_{B}\right)+\left(1-\mu_{a}\right)\left[-1+\gamma \hat{\mu}_{p}\left(1,0, \nu_{G}, \nu_{B}\right)\right]
\end{aligned}
$$

Especially, if $\gamma<\frac{\mu_{a}}{2-\mu_{a}}$, it cannot be $\nu_{G}^{*}=0$, since we have $\hat{\mu}_{p}(1,1)=\hat{\mu}_{p}(1,0)=0$ and $\hat{\mu}_{p}(0,1)=\hat{\mu}_{p}(0,0)=1$ (because $\left.\nu_{B}^{*}=0\right)$. Thus, that she will implement $x=1$ with a positive $\nu_{G}$.

\section{Claim 3}

Suppose that the good principal always chooses $x=0$ if $m=0$, and chooses $x=1$ with probability $\nu_{G}^{*} \in[0,1]$ if $m=1$. Also suppose that the biased principal chooses $x=1$ when $m=1$, and $x=0$ with probability $\nu_{B}^{*} \in[0,1)$ when $m=0$. Then, the good agent always sends $m=\theta$ and the biased agent always sends $m=1$.

Proof. 
Suppose that the agent observes $\theta=1$. Then, for the agent of both types, $x=1$ is the optimal decision. $x=0$ is chosen with probability $\left(1-\mu_{p}\right) \nu_{B}^{*}$ if he sends $m=0$, and $x=1$ is chosen with probability $\mu_{p} \nu_{G}^{*}+\left(1-\mu_{p}\right)$ when he sends $m=1$. Thus, both types send $m=1$ if $\theta=1$.

Next, suppose that the agent observes $\theta=0$. Then, $x=0$ is optimal for the good agent, and $x=1$ is optimal for the biased agent. Since $x=0$ is chosen with probability $\mu_{p}+\left(1-\mu_{p}\right) \nu_{B}^{*}$ if he sends $m=0$, and $x=1$ is chosen with probability $\mu_{p} \nu_{G}^{*}$ when he sends $m=1$, it is optimal for the good agent to send $m=0$. For the biased agent, the similar argument applies and he always send $m=1$ since the best decision for him is $x=1$.

Claim 1, 2, and 3 prove Proposition 1.

\section{Proof of Lemma 1.}

First of all, we define $\tilde{\mu}_{p}\left(x, \theta, \nu_{G}, \nu_{B}\right) \equiv \hat{\mu}_{p}\left(x, \theta, \nu_{G}, \nu_{B}, \hat{\alpha}^{G}, \hat{\alpha}^{B}\right)$, given the evaluator's beliefs on $\hat{\alpha}^{G}$ and $\hat{\alpha}^{B}$. Suppose that the biased principal receives a message $m=0$. Then, if she chooses $x=0$, her expected utility becomes

$$
-1+\gamma \tilde{\mu}_{p}\left(0,0, \nu_{G}, \nu_{B}\right)
$$

and if she chooses $x=1$, she obtains

$$
\gamma \tilde{\mu}_{p}\left(1,0, \nu_{G}, \nu_{B}\right)
$$

At any equilibrium such that $\nu_{B}^{* *}>0,-1+\gamma \tilde{\mu}_{p}\left(0,0, \nu_{G}, \nu_{B}\right) \leq \gamma \hat{\mu}_{p}\left(1,0, \nu_{G}, \nu_{B}\right)$ or

$$
\gamma\left[\tilde{\mu}_{p}\left(0,0, \nu_{G}^{* *}, \nu_{B}^{* *}\right)-\tilde{\mu}_{p}\left(1,0, \nu_{G}^{* *}, \nu_{B}^{* *}\right)\right] \leq 1
$$

must hold. Note $\tilde{\mu}_{p}\left(0,0, \nu_{G}, \nu_{B}\right)-\tilde{\mu}_{p}\left(1,0, \nu_{G}, \nu_{B}\right)$ is increasing in $\alpha^{G}$ and $\alpha^{B}$, but decreasing in $\nu_{B}$. Since $\nu_{B}^{*}$ corresponds to the solution where $\alpha_{G}=\alpha_{B}=1$, and 
$\nu_{B}^{*}>0$ satisfies $\gamma\left[\hat{\mu}_{p}\left(0,0, \nu_{G}^{*}, \nu_{B}^{*}\right)-\hat{\mu}_{p}\left(1,0, \nu_{G}^{*}, \nu_{B}^{*}\right)\right] \leq 1$, we must have $\nu_{B}^{* *} \leq \nu_{B}^{*}$. Furthermore, if $\gamma\left[\hat{\mu}_{p}\left(0,0, \nu_{G}^{*}, \nu_{B}^{*}\right)-\hat{\mu}_{p}\left(1,0, \nu_{G}^{*}, \nu_{B}^{*}\right)\right]=1$, i.e., $\nu_{B}^{*} \in(0,1)$, then in order to satisfy $\gamma\left[\tilde{\mu}_{p}\left(0,0, \nu_{G}^{* *}, \nu_{B}^{* *}\right)-\tilde{\mu}_{p}\left(1,0, \nu_{G}^{* *}, \nu_{B}^{* *}\right)\right]=1$ for $\alpha^{B}<1, \nu_{B}^{* *}<\nu_{B}^{*}$ must be the case.

\section{Proof of Proposition 3.}

Suppose that the evaluator believes that the biased principal keeps the authority with probability of $\hat{\alpha}^{B}$. Then, the biased principal's expected payoff when keeping authority (i.e., $\alpha^{B}=1$ ) becomes

$$
\begin{gathered}
\frac{1}{2}\left\{\mu_{a}\left[\nu_{B}^{* *}\left(-1+\gamma \tilde{\mu}_{p}\left(0,0, \nu_{G}^{* *}, \nu_{B}^{* *}\right)\right)+\left(1-\nu_{B}^{* *}\right) \gamma \tilde{\mu}_{p}\left(1,0, \nu_{G}^{* *}, \nu_{B}^{* *}\right)\right]\right. \\
\left.+\left(1-\mu_{a}\right) \gamma \tilde{\mu}_{p}\left(1,0, \nu_{G}^{* *}, \nu_{B}^{* *}\right)\right\}+\frac{1}{2} \gamma \tilde{\mu}_{p}\left(1,1, \nu_{G}^{* *}, \nu_{B}^{* *}\right)
\end{gathered}
$$

On the other hand, when the biased principal delegates the authority (i.e., $\alpha^{B}=0$ ), her expected utility is

$$
\frac{1}{2}\left\{\mu_{a}\left(-1+\gamma \tilde{\mu}_{p}\left(0,0, \nu_{G}^{* *}, \nu_{B}^{* *}\right)\right)+\left(1-\mu_{a}\right) \gamma \tilde{\mu}_{p}\left(1,0, \nu_{G}^{* *}, \nu_{B}^{* *}\right)\right\}+\frac{1}{2} \gamma \tilde{\mu}_{p}\left(1,1, \nu_{G}^{* *}, \nu_{B}^{* *}\right) .
$$

The biased principal keeps the authority if

$$
\gamma \tilde{\mu}_{p}\left(1,0, \nu_{G}^{* *}, \nu_{B}^{* *}\right) \geq-1+\gamma \tilde{\mu}_{p}\left(0,0, \nu_{G}^{* *}, \nu_{B}^{* *}\right) .
$$

Since $\nu_{B}^{* *} \leq \nu_{B}^{*}, \nu_{B}^{* *}<1$ as well. As in $(20), \nu_{B}^{* *} \in(0,1)$ if and only if $\gamma \tilde{\mu}_{p}\left(1,0, \nu_{G}^{* *}, \nu_{B}^{* *}\right)=-1+\gamma \tilde{\mu}_{p}\left(0,0, \nu_{G}^{* *}, \nu_{B}^{* *}\right)$.

Suppose that $\nu_{B}^{* *}>0$ holds for a given $\hat{\alpha}^{B}$, from (21) and (22), we can see that keeping the authority and delegating to the agent is indifferent for the biased principal since $\gamma \tilde{\mu}_{p}\left(1,0, \nu_{G}^{* *}, \nu_{B}^{* *}\right)=-1+\gamma \tilde{\mu}_{p}\left(0,0, \nu_{G}^{* *}, \nu_{B}^{* *}\right)$. Then, the biased principal can choose optimally $\alpha^{B *}=\hat{\alpha}^{B} \in(0,1)$. 
However, $\hat{\alpha}^{B}=0$ cannot be supported as the equilibrium since $\nu_{B}=0$ at $\hat{\alpha}^{B}=0$, which implies that (21) is strictly greater than (22) (since in this case, $\left.\hat{\mu}_{p}(0,0, \nu, 0)=\hat{\mu}_{p}(1,0, \nu, 0)=\mu_{p}\right)$, so that $\hat{\alpha}^{B}$ should be 1 .

Next, if $\nu_{B}^{* *}=0$ for a given $\hat{\alpha}^{B}$, then $\tilde{\mu}_{p}\left(1,0, \nu_{G}^{* *}, \nu_{B}^{* *}\right)>-1+\gamma \tilde{\mu}_{p}\left(0,0, \nu_{G}^{* *}, \nu_{B}^{* *}\right)$ must be the case. It follows that (21) is always greater than (22), and then the biased principal chooses $\alpha^{B *}=1$. Hence, as long as $\alpha^{B *}<1, \nu_{B}^{* *}>0$ must be the case.

\section{Proof of Proposition 4.}

For the good principal, her expected payoff when keeping authority (i.e., $\alpha^{G}=1$ ) is

$$
\begin{aligned}
& \frac{1}{2}\left\{\mu_{a} \gamma \tilde{\mu}_{p}\left(0,0, \nu_{G}^{* *}, \nu_{B}^{* *}\right)+\left(1-\mu_{a}\right)\left[\left(1-\nu_{G}\right) \gamma \tilde{\mu}_{p}\left(0,0, \nu_{G}^{* *}, \nu_{B}^{* *}\right)+\nu_{G}\left(-1+\tilde{\mu}_{p}\left(1,0, \nu_{G}^{* *}, \nu_{B}^{* *}\right)\right)\right]\right\} \\
+ & \frac{1}{2}\left\{\nu_{G} \gamma \tilde{\mu}_{p}\left(1,1, \nu_{G}^{* *}, \nu_{B}^{* *}\right)+\left(1-\nu_{G}\right)\left(-1+\gamma \tilde{\mu}_{p}\left(0,1, \nu_{G}^{* *}, \nu_{B}^{* *}\right)\right)\right\}
\end{aligned}
$$

When she delegates the authority (i.e., $\alpha^{G}=0$ ), her expected utility is

$$
\frac{1}{2}\left[\mu_{a} \gamma \tilde{\mu}_{p}\left(0,0, \nu_{G}^{* *}, \nu_{B}^{* *}\right)+\left(1-\mu_{a}\right)\left(-1+\gamma \tilde{\mu}_{p}\left(1,0, \nu_{G}^{* *}, \nu_{B}^{* *}\right)\right)\right]+\frac{1}{2} \gamma \tilde{\mu}_{p}\left(1,1, \nu_{G}^{* *}, \nu_{B}^{* *}\right)
$$

The good principal keeps the authority if

$$
\begin{aligned}
& \left(1-\nu_{G}^{* *}\right)\left\{\left[-1+\gamma \tilde{\mu}_{p}\left(0,1, \nu_{G}^{* *}, \nu_{B}^{* *}\right)\right]+\left(1-\mu_{a}\right) \gamma \tilde{\mu}_{p}\left(0,0, \nu_{G}^{* *}, \nu_{B}^{* *}\right)\right\} \\
\geq & \left(1-\nu_{G}^{* *}\right)\left\{\tilde{\mu}_{p}\left(1,1, \nu_{G}^{* *}, \nu_{B}^{* *}\right)+\left(1-\mu_{a}\right)\left[-1+\gamma \tilde{\mu}_{p}\left(1,0, \nu_{G}^{* *}, \nu_{B}^{* *}\right)\right]\right\}
\end{aligned}
$$

If $\nu_{G}^{* *}=1$, the good principal is indifferent between keeping and delegating the authority. If $\alpha^{G *} \in(0,1), \nu_{G}^{* *} \in(0,1)$. On the other hand, if $\alpha^{G *}=1$ then $\nu_{G}^{* *}=0$.

Since $\tilde{\mu}_{p}\left(0,0, \nu_{G}^{* *}, \nu_{B}^{* *}\right)$ increases in $\hat{\alpha}^{G}$ and decreases in $\nu_{G}, \tilde{\mu}_{p}\left(1,1, \nu_{G}^{* *}, \nu_{B}^{* *}\right)$ and $\tilde{\mu}_{p}\left(1,0, \nu_{G}^{* *}, \nu_{B}^{* *}\right)$ decrease in $\hat{\alpha}^{G}$ and increase in $\nu_{G}$, if $\alpha^{G *}<1$ then $\nu_{G}^{* *}<\nu_{G}^{*}$. 


\section{Appendix B: Generalized Proposition 1}

In this appendix, we want to show Proposition 1 by considering all possible cases. Let $\nu_{G}(m)$ be the probability that the good principal chooses $x=0$ when she receives message $m$ from the agent. Similarly, let $\nu_{B}(m)$ be that for the biased principal. The following proposition characterizes the properties of the principal's equilibrium strategy under the principal's discretion.

\section{Proposition $1^{*}$}

Under the principal's discretion, the equilibrium satisfies:

1. The good agent sends $m=\theta$ always.

2. The biased agent sends $m=1$ always.

3. The good principal's equilibrium strategy satisfies $\nu_{G}(0)=1$ and $\nu_{G}(1) \in[0,1]$.

4. The biased principal's equilibrium strategy satisfies $\nu_{B}(0) \leq \nu_{G}(0)=1$ and $\nu_{B}(1) \leq \nu_{G}(1)$.

Proof.

First of all, we distinguish three kinds of equilibria as follows:

1. Most informative equilibrium: the good principal's strategy satisfies $\nu_{G}(0)=1$ and $\nu_{G}(1)=0$, and the biased principal's strategy satisfies $\nu_{B}(0) \in[0,1)$ and $\nu_{B}(1)=0$.

2. Pooling equilibrium (uninformative equilibrium): $\nu_{G}(m)=\nu_{B}(m)=1$ for $m=0,1$.

3. Informative equilibrium: $\nu_{G}(0)=1$ and $\nu_{G}(1) \in(0,1)$, and $\nu_{B}(0)<\nu_{G}(0)=1$ and $\nu_{B}(1) \leq \nu_{G}(1)$. 
Whether an equilibrium is informative or not depends upon a level of $\gamma$.

Suppose that the good agent sends $m=\theta$ and the biased agent sends $m=1$ always. Then, the principal has beliefs $\operatorname{Prob}\{\theta=0 \mid m=0\}=1$, and $\operatorname{Prob}\{\theta=$ $1 \mid m=1\}=\frac{1}{1+\left(1-\mu_{a}\right)}>\frac{1}{2}$. To prove Proposition $1^{*}$, we make several claims as follows.

\section{Most Informative Equilibrium}

\section{Claim 4}

Suppose that the good agent always sends $m=\theta$ and the biased agent sends $m=1$ always. Then, there can be the equilibrium under which (i) the good principal makes decision $x=m$ (correct decision), and (ii) the biased principal chooses $x=1$ when $m=1$, and $x=1$ with probability $\nu_{B}(0) \in[0,1)$ when $m=0$.

\section{Proof.}

First of all, suppose that the good principal always chooses $x=m$. Then the biased principal chooses $x=1$ when $m=1$. Suppose not. By choosing $x=1$, the biased principal can increase her reputation (since the good principal chooses $x=1$ if $m=1$ ) and induce $x=1$. Suppose that the biased principal receives $m=0$. If she implements $x=0$, then her expected utility becomes $-1+\gamma \hat{\mu}_{p}(0,0, \nu)$. If she implements $x=1$, her expected utility is $\gamma \hat{\mu}_{p}(1,0, \nu)$. We can easily see for any value of $\nu,-1+\gamma \hat{\mu}_{p}(0,0, \nu) \leq \gamma \hat{\mu}_{p}(1,0, \nu)$ holds. Therefore, $\nu^{*}<1$. If $-1+\gamma \hat{\mu}_{p}(0,0, \nu)=\gamma \hat{\mu}_{p}(1,0, \nu)$, then some $\nu^{*} \in(0,1)$ is the equilibrium. If $-1+$ $\gamma \hat{\mu}_{p}(0,0, \nu)<\gamma \hat{\mu}_{p}(1,0, \nu)$ for every value of $\nu$, then $\nu^{*}=0$ is the equilibrium.

Next, suppose that the good principal chooses $x=m$ on the equilibrium path. Thus, neither type of the principal chooses $x=0$ when $m=1$. That is, $(x, \theta)=(0,1)$ is off the equilibrium path. We assume that $\hat{\mu}_{p}(0,1, \nu)=\mu_{p}$ is the off-equilibrium belief for this event. Given this, by choosing $x=0$ when $m=1$, the good principal 
obtains the expected utility $\frac{1}{2}\left[-1+\gamma \mu_{p}\right]+\frac{1}{2}\left(1-\mu_{a}\right) \gamma \hat{\mu}_{p}(0,0, \nu)$. If she chooses $x=1$ instead, her expected utility is $\frac{1}{2} \gamma \hat{\mu}_{p}(1,1, \nu)+\frac{1}{2}\left(1-\mu_{a}\right)\left[-1+\gamma \hat{\mu}_{p}(1,0, \nu)\right]$, where $\hat{\mu}_{p}(1,1)=\mu_{p}$. Thus, if $\mu_{a}+\left(1-\mu_{a}\right) \gamma\left[\hat{\mu}_{p}(1,0, \nu)-\hat{\mu}_{p}(0,0, \nu)\right]>0$, the good principal chooses $x=1$ when $m=1$.

\section{Pooling Equilibrium}

\section{Claim 5}

Suppose that the good agent always sends $m=\theta$ and the biased agent sends $m=1$ always. Then, there can be the equilibrium under which either type of the principal chooses $x=0$.

Proof.

Under the pooling equilibrium, the ex post reputation always coincides with the ex ante one, that is $\hat{\mu}_{p}=\mu_{p}$. Now suppose that the biased principal receives $m=0$. If she implements $x=0$, then her expected utility becomes $-1+\gamma \mu_{p}$. If she implements $x=1$, her expected utility is $\gamma \tilde{\mu}_{p}$, where $\tilde{\mu}_{p}$ is the off the equilibrium path belief. Thus, as long as $-1+\gamma \mu_{p} \geq \gamma \tilde{\mu}_{p}$ is satisfied, the biased principal chooses $x=0$ if $m=0$. We assume $\tilde{\mu}_{p}=0$ as the off equilibrium belief. Then, if $\gamma$ is sufficiently large, the biased agent chooses $x=0$ if $m=0$. Similar argument applies if the biased principal receives $m=1$.

Next, suppose that the good principal receives $m=0$. In this case, she has no incentive to choose $x=1$ with $\tilde{\mu}_{p}=0$ since $\gamma \mu_{p}>-1$ always. If the good principal receives $m=1$, then, by choosing $x=0$, her utility becomes $-1+\gamma \mu_{p}$, and becomes $\gamma \tilde{\mu}_{p}$ if $x=1$. Thus, as in the case of the biased principal, if $\gamma$ is sufficiently large, then the good principal chooses $x=0$. 
Informative Equilibrium

Besides the most informative equilibrium and the pooling equilibrium, any equilibrium can be characterized by the following claim.

\section{Claim 6}

Suppose that the good agent always sends $m=\theta$ and the biased agent sends $m=1$ always. Then, any equilibrium strategy which is not most informative or pooling satisfies (i) $\nu_{G}(0)=1$ and $\nu_{G}(1) \in(0,1]$, (ii) $\nu_{B}(0) \in[0,1)$ and $\nu_{B}(1) \in(0,1)$, and (iii) $\nu_{B}(0)<\nu_{G}(0)$ and $\nu_{B}(1) \leq \nu_{G}(1)$.

\section{Proof.}

The idea of thte proof is basically similar to Morris (2001). Let $\hat{\mu}_{p}\left(x, \theta, \nu_{B}(m), \nu_{G}(m)\right)$ be the ex post belief on the principal's type for given $\nu_{B}(m)$ and $\nu_{G}(m)$. Then,

$$
\begin{gathered}
\hat{\mu}_{p}\left(1,1, \nu_{B}(m), \nu_{G}(m)\right)=\frac{\mu_{p}\left(1-\nu_{G}(1)\right)}{\mu_{p}\left(1-\nu_{G}(1)\right)+\left(1-\mu_{p}\right)\left(1-\nu_{B}(1)\right)} \\
\hat{\mu}_{p}\left(0,1, \nu_{B}(m), \nu_{G}(m)\right)=\frac{\mu_{p} \nu_{G}(1)}{\mu_{p} \nu_{G}(1)+\left(1-\mu_{p}\right) \nu_{B}(1)} \\
\frac{\hat{\mu}_{p}\left(1,0, \nu_{B}(m), \nu_{G}(m)\right)=}{\mu_{a}\left[\mu_{p}\left(1-\nu_{G}(0)\right)+\left(1-\mu_{p}\right)\left(1-\nu_{B}(0)\right)\right]+\left(1-\mu_{a}\right)\left[\mu_{p}\left(1-\nu_{G}(1)\right)+\left(1-\mu_{p}\right)\left(1-\nu_{B}(1)\right)\right]} \\
\hat{\mu}_{p}\left(0,0, \nu_{B}(m), \nu_{G}(m)\right)=\frac{\mu_{a} \mu_{p}\left(1-\nu_{p}(0)\right)+\left(1-\mu_{G}(0)+\left(1-\mu_{a}\right) \mu_{p} \nu_{G}(1)\right.}{\mu_{a}\left[\mu_{p} \nu_{G}(0)+\left(1-\mu_{p}\right) \nu_{B}(0)\right]+\left(1-\mu_{a}\right)\left[\mu_{p} \nu_{G}(1)+\left(1-\mu_{p}\right) \nu_{B}(1)\right]}
\end{gathered}
$$

Step $1 \hat{\mu}_{p}\left(1,1, \nu_{B}(m), \nu_{G}(m)\right)=\hat{\mu}_{p}\left(0,1, \nu_{B}(m), \nu_{G}(m)\right)=\hat{\mu}_{p}\left(1,0, \nu_{B}(m), \nu_{G}(m)\right)=$ $\hat{\mu}_{p}\left(0,0, \nu_{B}(m), \nu_{G}(m)\right)$ is a pooling equilibrium and is therefore neglected.

Step $2 \hat{\mu}_{p}\left(0,1, \nu_{B}(m), \nu_{G}(m)\right) \geq \hat{\mu}_{p}\left(1,1, \nu_{B}(m), \nu_{G}(m)\right)$. 
Suppose that $\hat{\mu}_{p}\left(0,1, \nu_{B}(m), \nu_{G}(m)\right)<\hat{\mu}_{p}\left(1,1, \nu_{B}(m), \nu_{G}(m)\right)$. Then, the biased principal has no incentive to choose $x=0$ when $m=1$, and $\nu_{B}(1)=0$. In this case, if $\nu_{G}(1)=0, \hat{\mu}_{p}\left(1,1, \nu_{B}(m), \nu_{G}(m)\right)=\mu_{p}$, and the equilibrium becomes the most informative equilibrium. If $\nu_{G}(1)>0, \hat{\mu}_{p}\left(0,1, \nu_{B}(m), \nu_{G}(m)\right) \geq$ $\hat{\mu}_{p}\left(1,1, \nu_{B}(m), \nu_{G}(m)\right)$. Contradiction.

Step $3 \hat{\mu}_{p}\left(0,0, \nu_{B}(m), \nu_{G}(m)\right) \geq \hat{\mu}_{p}\left(1,0, \nu_{B}(m), \nu_{G}(m)\right)$.

Suppose that $\hat{\mu}_{p}\left(0,0, \nu_{B}(m), \nu_{G}(m)\right)<\hat{\mu}_{p}\left(1,0, \nu_{B}(m), \nu_{G}(m)\right)$. Then, the biased principal has no incentive to choose $x=0$ when $m=0$, and $\nu_{B}(0)=0$. In this case, the good principal has a strong incentive to choose $x=0$ when $m=0$ since $\hat{\mu}_{p}\left(0,1, \nu_{B}(m), \nu_{G}(m)\right)>\hat{\mu}_{p}\left(1,1, \nu_{B}(m), \nu_{G}(m)\right)$ as long as $\nu_{G}(0)>0$. Contradiction.

Step $4 \nu_{G}(0)=1$.

Clearly, $\hat{\mu}_{p}\left(0,0, \nu_{B}(m), \nu_{G}(m)\right) \geq \hat{\mu}_{p}\left(1,0, \nu_{B}(m), \nu_{G}(m)\right)$.

Step $5 \hat{\mu}_{p}\left(1,1, \nu_{B}(m), \nu_{G}(m)\right) \geq \hat{\mu}_{p}\left(1,0, \nu_{B}(m), \nu_{G}(m)\right)$, and $\nu_{G}(1) \leq \nu_{G}(0)=1$.

Note that $\nu_{G}(0)=1$. Then, straight calculations give us $\hat{\mu}_{p}\left(1,1, \nu_{B}(m), \nu_{G}(m)\right)$ $\geq \hat{\mu}_{p}\left(1,0, \nu_{B}(m), \nu_{G}(m)\right)$. Also, this inequality implies $\nu_{G}(1) \leq \nu_{G}(0)=1$.

Step $6 \hat{\mu}_{p}\left(0,1, \nu_{B}(m), \nu_{G}(m)\right) \geq \hat{\mu}_{p}\left(0,0, \nu_{B}(m), \nu_{G}(m)\right)$.

Suppose not. Then, we have $\hat{\mu}_{p}\left(0,1, \nu_{B}(m), \nu_{G}(m)\right)<\hat{\mu}_{p}\left(0,0, \nu_{B}(m), \nu_{G}(m)\right)$. Note that $\nu_{G}(1) \leq \nu_{G}(0)=1$. Then, in order to satisfy above inequality, we must have $\nu_{B}(1) \leq \nu_{B}(0)$. But this contradicts to $\hat{\mu}_{p}\left(0,0, \nu_{B}(m), \nu_{G}(m)\right) \geq$ $\hat{\mu}_{p}\left(1,0, \nu_{B}(m), \nu_{G}(m)\right)$.

Step $7 \nu_{B}(0)<\nu_{G}(0)=1$.

The argument is similar to Claim 1. 
Step $8 \nu_{B}(1) \leq \nu_{G}(1)$.

Suppose not. Then $\nu_{B}(1)>\nu_{G}(1)$. But it contradicts to $\hat{\mu}_{p}\left(0,1, \nu_{B}(m), \nu_{G}(m)\right)$ $\geq \hat{\mu}_{p}\left(1,1, \nu_{B}(m), \nu_{G}(m)\right)$.

\section{Claim 7}

The good agent always sends $m=\theta$ and the biased agent always sends $m=1$ in equilibrium.

\section{Proof.}

Suppose that the agent observes $\theta=1$. Then, for the agent of both types, $x=1$ is the optimal decision. Since the principal chooses $x=1$ with positive probability when $m=1$ except in the pooling equilibrium, and chooses $x=0$ more often when $m=0$ than $m=1$, both types send $m=1$ if $\theta=1$. In the pooling equilibrium, the agent's message cannot affect on the principal's decision making, and thus the agent of both types can send $m=1$ on the equilibrium path.

Next, suppose that the agent observes $\theta=0$. Then, $x=0$ is optimal for the good agent, and $x=1$ is optimal for the biased agent. Since $x=0$ is chosen with probability $\mu_{p}+\left(1-\mu_{p}\right) \nu_{B}(0)$ if he sends $m=0$, and $x=0$ is chosen with probability of $\mu_{p} \nu_{G}(1)+\left(1-\mu_{p}\right) \nu_{B}(1)<\mu_{p}+\left(1-\mu_{p}\right) \nu_{B}(0)$ when he sends $m=1$, it is optimal for the good agent to send $m=0$. For the biased agent, the similar argument applies and he always send $m=1$ since the best decision for him is $x=1$.

Claim 4, 5, 6 and 7 prove Proposition $1^{*}$. 